\title{
Exact Solutions of Equation Generated by the Jaulent-Miodek Hierarchy by $\left(G^{\prime} / G\right)$-Expansion Method
}

\author{
Wafaa M. Taha and M. S. M. Noorani \\ Pusat Pengajian Sains Matematik, Universiti Kebangsaan Malaysia, 43600 UKM Bangi, Selangor, Malaysia
}

Correspondence should be addressed to Wafaa M. Taha; wafaa_y2005@yahoo.com

Received 30 September 2012; Accepted 28 December 2012

Academic Editor: Farzad Khani

Copyright (c) 2013 W. M. Taha and M. S. M. Noorani. This is an open access article distributed under the Creative Commons Attribution License, which permits unrestricted use, distribution, and reproduction in any medium, provided the original work is properly cited.

\begin{abstract}
The $\left(G^{\prime} / G\right)$-expansion method is proposed for constructing more general exact solutions of the nonlinear $(2+1)$-dimensional equation generated by the Jaulent-Miodek Hierarchy. As a result, when the parameters are taken at special values, some new traveling wave solutions are obtained which include solitary wave solutions which are based from the hyperbolic functions, trigonometric functions, and rational functions. We find in this work that the $\left(G^{\prime} / G\right)$-expansion method give some new results which are easier and faster to compute by the help of a symbolic computation system. The results obtained were compared with tanh method.
\end{abstract}

\section{Introduction}

In the present paper, we obtained exact solutions of the nonlinear $(2+1)$-dimensional equation (1) generated by the Jaulent-Miodek Hierarchy [1] in the form

$$
w_{t}=-\frac{1}{4}\left(w_{x x}-2 w^{3}\right)_{x}-\frac{3}{4}\left(\frac{1}{4} \partial_{x}^{-1} w_{y y}+w_{x} \partial_{x}^{-1} w_{y}\right),
$$

where $y$ is a spatial variable, and $w(x, y, t)$ is the analytic function with respect to $x, y$, and $t$. In addition, $\partial_{x}^{-1}$ is the inverse of $\partial_{x}$ with $\partial_{x} \partial_{x}^{-1}=\partial_{x}^{-1} \partial_{x}=1$ and $\left(\partial_{x}^{-1} f\right)(x)=$ $\int_{-\infty}^{x} f(t) d t$, under the decaying condition at infinity. This equation was studied by Liu and Yan by using qualitative analysis method [2]. It is clear that searching for explicit solutions of nonlinear evolution equation (NLEEs) by using various method has become the main aim for many authors. Many powerful methods have been created and developed to obtain analytic solutions of (NLEEs), such as the the tanhcoth method [3-5], sine-cosine method [6], homogeneous balance method [7], exp-function method [8, 9], Hirota bilinear transformation [10, 11], Jacobi elliptic function method [12], and solitary wave ansatz method [13]. One of the most effective direct method to build traveling wave solution of (NLEEs) is the $\left(G^{\prime} / G\right)$-expansion method, which was first proposed by Wang et al. [14]. It is assumed that the traveling wave solutions can be expressed by a polynomial in $\left(G^{\prime} / G\right)$, where $G=G(\zeta)$ satisfies the following second-order linear ordinary differential equation $G^{\prime \prime}(\zeta)+\lambda G^{\prime}(\zeta)+\mu G(\zeta)=0$, where $\zeta=x-c t$, and $\lambda, \mu$, and $c$ are constants. Next, Bekir applied the method to some nonlinear evolution equations earning some traveling wave solutions [15]. After that, Zhang et al. have improved and generalized the $\left(G^{\prime} / G\right)$-expansion method to seek exact solutions of other nonlinear evolution equations [16]. Moreover, Zhang et al. have further extended the method to deal with evolution equations with variable coefficients [17]. In fact the $\left(G^{\prime} / G\right)$-expansion method has been successfully applied to obtain exact solution for a variety of (NLEEs) [12, 18-35]. In this paper, the $\left(G^{\prime} / G\right)$ expansion method is proposed for constructing more general exact solutions of the above nonlinear $(2+1)$-dimensional equation generated by the Jaulent-Miodek Hierarchy. Our paper is organized as follows: in Section 2, the description of the $\left(G^{\prime} / G\right)$-expansion method is provided; in Section 3, we spell out the applications of this method to the nonlinear $(2+1)$-dimensional equation; in Section 4, application of the tanh method on the nonlinear $(2+1)$-dimensional equation and finally in Section 5, some conclusions are given. 


\section{Description the $\left(G^{\prime} / G\right)$-Expansion Method}

In this section we describe the $\left(G^{\prime} / G\right)$-expansion method for finding traveling wave solutions of (NLEEs). Suppose that a nonlinear evolution equation, say in three independent variables $x, y$, and $t$, is given by

$$
p\left(u, u_{t}, u_{x}, u_{y}, u_{x t}, u_{y t}, u_{t t}, u_{x x}, u_{y y}, \ldots\right)=0,
$$

where $u=u(x, y, t)$ is an unknown function, and $p$ is a polynomial in $u=u(x, y, t)$ and its various partial derivatives, in which highest-order derivatives and nonlinear terms are involved. To determine the $\left(G^{\prime} / G\right)$-expansion method, we take the following six steps.

Step 1. To find the traveling wave solutions of (2), we introduce the wave variable

$$
u(x, y, t)=u(\zeta), \quad \zeta=x+y+c t,
$$

where $c$ is constant. Substituting (3) into (2), we obtain the following ordinary differential equations (ODE):

$$
p\left(u, c u^{\prime}, u^{\prime}, c u^{\prime \prime}, c^{2} u^{\prime \prime}, u^{\prime \prime}, \ldots\right)=0 .
$$

Step 2. If necessary we integrate (4) as many times as possible and set the constants of integration to be zero for simplicity.

Step 3. We suppose the solution of nonlinear partial differential equation can be expressed by a polynomial in $\left(G^{\prime} / G\right)$ as

$$
u(\zeta)=\sum_{i=0}^{m} a_{i}\left(\frac{G^{\prime}}{G}\right)^{i}
$$

where $G=G(\zeta)$ satisfies the second-order linear ordinary differential equation

$$
G^{\prime \prime}(\zeta)+\lambda G^{\prime}(\zeta)+\mu G(\zeta)=0,
$$

where $G^{\prime}=d G / d \zeta, G^{\prime \prime}=d^{2} G / d \zeta^{2}$, and $a_{i}, \lambda$, and $\mu$ are real constants with $a_{m} \neq 0$.

Step 4 . The positive integer $m$ can be determined by considering the homogeneous balance between the highest-order derivatives and nonlinear terms appearing in (4) as follows: if we define the degree of $u(\zeta)$ as $D[u(\zeta)]=m$, then the degree of other expressions is defined by

$$
D\left[u^{r}\left(\frac{d^{q} u}{d \zeta^{q}}\right)^{s}\right]=m r+s(q+m) .
$$

Therefore, we can get the value of $m$ in (5).

Step 5. Substituting (5) into (4), using (6), collecting all terms with the same order of $\left(G^{\prime} / G\right)$ together, and then setting each coefficient of this polynomial to zero yield a set of algebraic equations for $a_{i}, c, \lambda$, and $\mu$.

Step 6. Substituting $a_{i}, c, \lambda$, and $\mu$ obtained in Step 5 and the general solutions of (6) into (5), we obtain traveling wave solutions of the nonlinear (PDE).

\section{Application the $\left(G^{\prime} / G\right)$-Expansion Method}

3.1. Equation Generated by the Jaulent-Miodek Hierarchy. In this section, the $\left(G^{\prime} / G\right)$-expansion method will be applied to the equation generated by the Jaulent-Miodek Hierarchy in the form

$$
w_{t}=-\frac{1}{4}\left(w_{x x}-2 w^{3}\right)_{x}-\frac{3}{4}\left(\frac{1}{4} \partial_{x}^{-1} w_{y y}+w_{x} \partial_{x}^{-1} w_{y}\right) .
$$

We first remove the integral term in (8) by introducing the potential

$$
w(x, y, t)=u_{x}(x, y, t)
$$

to carry (8) to the equation

$$
u_{x t}+\frac{1}{4} u_{x x x x}-\frac{3}{2} u_{x}^{2} u_{x x}+\frac{3}{16} u_{y y}+\frac{3}{4} u_{x x} u_{y}=0 .
$$

Let $u(x, y, t)=u(\zeta), \zeta=x+y+c t$, where $c$ is the wave speed. Substituting the above traveling wave variable $\zeta=x+y+c t$ in to (10) yields

$$
c u^{\prime \prime}+\frac{1}{4} u^{\prime \prime \prime \prime}-\frac{3}{2}\left(u^{\prime}\right)^{2} u^{\prime \prime}+\frac{3}{16} u^{\prime \prime}+\frac{3}{4} u^{\prime \prime} u^{\prime}=0 .
$$

Integrating (11) above with respect to $\zeta$ once yields

$$
16 c u^{\prime}+4 u^{\prime \prime \prime}-8\left(u^{\prime}\right)^{3}+3 u^{\prime}+6\left(u^{\prime}\right)^{2}=0 .
$$

For simplicity, the integration constant is taken as zero. Assume that

$$
u^{\prime}=v \text {. }
$$

Substituting (13) into (12), we obtain ordinary differential equation as follows:

$$
16 c v+4 v^{\prime \prime}-8 v^{3}+3 v+6 v^{2}=0 .
$$

We suppose that the solution of (14) has the following formal solution:

$$
v(\zeta)=\sum_{i=0}^{m} a_{i}\left(\frac{G^{\prime}}{G}\right)^{i}
$$

where $G=G(\zeta)$ satisfies the second-order linear ordinary differential equation $G^{\prime \prime}(\zeta)+\lambda G^{\prime}(\zeta)+\mu G(\zeta)=0$.

According to Step $4 m=1$, and therefore (15) become

$$
v(\zeta)=a_{0}+a_{1}\left(\frac{G^{\prime}}{G}\right), \quad a_{1} \neq 0 .
$$

On substituting (16) along with (6) into (14), collecting all terms with the same powers of $\left(G^{\prime} / G\right)$, and setting 
each cofficient to zero yield a set of simultaneous algebraic equations for $a_{0}, a_{1}, c, \lambda$, and $\mu$ as follows:

$$
\begin{aligned}
& \left(\frac{G^{\prime}}{G}\right)^{0}: 16 c a_{0}+4 a_{1} \lambda \mu-8 a_{0}^{3}+3 a_{0}+6 a_{0}^{2}=0 \\
& \left(\frac{G^{\prime}}{G}\right)^{1}: 16 c a_{1}+4\left(a_{1} \lambda^{2}+2 a_{1} \mu\right) \\
& \quad-24 a_{0}^{2} a_{1}+3 a_{1}+12 a_{0} a_{1}=0 \\
& \left(\frac{G^{\prime}}{G}\right)^{2}: 12 a_{1} \lambda-24 a_{0} a_{1}^{2}+6 a_{1}^{2}=0 \\
& \left(\frac{G^{\prime}}{G}\right)^{3}: 8 a_{1}-8 a_{1}^{3}=0
\end{aligned}
$$

Solving this set of algebraic equations by Maple or Mathematica, we can obtain the following results:

$$
\text { first solution set: } a_{0}=\frac{1}{2} \lambda+\frac{1}{4} \text {, }
$$

$$
\begin{aligned}
& a_{1}=1, \\
& c=-\frac{1}{4}, \\
& \mu=-\frac{1}{16}+\frac{1}{4} \lambda^{2},
\end{aligned}
$$$$
\text { second solution set: } a_{0}=-\frac{1}{2} \lambda+\frac{1}{4} \text {, }
$$

$$
\begin{aligned}
& a_{1}=-1, \\
& c=-\frac{1}{4}, \\
& \mu=-\frac{1}{16}+\frac{1}{4} \lambda^{2},
\end{aligned}
$$

where $\lambda$ is arbitrary constant. By using (18), expression (16) can be written as

$$
\begin{gathered}
v(\zeta)=\frac{1}{2} \lambda+\frac{1}{4}+\left(\frac{G^{\prime}}{G}\right), \\
v(\zeta)=-\frac{1}{2} \lambda+\frac{1}{4}-\left(\frac{G^{\prime}}{G}\right),
\end{gathered}
$$

where $\zeta=x+y-(1 / 4) t$.

Equation (19) is the formula of a solution of (8).

3.2. First Traveling Wave Solution Set. Substituting the general solutions of (6) into (19) we have three types of traveling wave solutions of the equation generated by the JaulentMiodek Hierarchy as follows.
Case a. When $\lambda^{2}-4 \mu>0$, we obtain the hyperbolic function solutions of (8)

$$
\begin{aligned}
& v(\zeta) \\
& =\frac{1}{2} \lambda+\frac{1}{4} \\
& +\left[\frac{-\lambda}{2}+\frac{\sqrt{\lambda^{2}-4 \mu}}{2}\right. \\
& \left.+\left(\frac{c_{1} \sinh \left\{\left(\sqrt{\lambda^{2}-4 \mu} / 2\right) \zeta\right\}+c_{2} \cosh \left\{\left(\sqrt{\lambda^{2}-4 \mu} / 2\right) \zeta\right\}}{c_{1} \cosh \left\{\left(\sqrt{\lambda^{2}-4 \mu} / 2\right) \zeta\right\}+c_{2} \sinh \left\{\left(\sqrt{\lambda^{2}-4 \mu} / 2\right) \zeta\right\}}\right)\right],
\end{aligned}
$$

where $\lambda, c_{1}$, and $c_{2}$ are arbitrary constants, and $\zeta=x+y-$ $(1 / 4) t$.

In particular, if $c_{1}$ and $c_{2}$ are taken as special values, the various known results in the literature can be rediscovered; for instance, when setting $c_{1}=0, c_{2}=1$ and $\mu=-1 / 16+$ $(1 / 4) \lambda^{2}, v$ becomes

$$
v_{1}(\zeta)=0.25+0.25 \operatorname{coth}\{0.25 \zeta\},
$$

which is the solitary wave solution of (8).

If we take $c_{2}=0, c_{1}=1$ and $\mu=-1 / 16+(1 / 4) \lambda^{2}$ above, then $v$ becomes

$$
v_{2}(\zeta)=0.25+0.25 \tanh \{0.25 \zeta\},
$$

and more solitary wave solutions of (8) can be obtained.

Biswas and Kara in (2010) found 1-soliton solution of the Jaulent-Miodek equation with power law nonlinearity that is derived by solitary wave ansatz method [13].

Case $b$. When $\lambda^{2}-4 \mu<0$, we get the trigonometric function solutions of (8) as follows:

$$
\begin{aligned}
& v(\zeta) \\
& =\frac{1}{2} \lambda+\frac{1}{4} \\
& +\left[\frac{-\lambda}{2}+\frac{\sqrt{4 \mu-\lambda^{2}}}{2}\right. \\
& \quad \times\left(\frac{\left.\left.-c_{1} \sin \left\{\left(\sqrt{4 \mu-\lambda^{2}} / 2\right) \zeta\right\}+c_{2} \cos \left\{\left(\sqrt{4 \mu-\lambda^{2}} / 2\right) \zeta\right\}\right)\right],}{\left.c_{1} \cos \left\{\left(\sqrt{4 \mu-\lambda^{2}} / 2\right) \zeta\right\}+c_{2} \sin \left\{\left(\sqrt{4 \mu-\lambda^{2}} / 2\right) \zeta\right\}\right)},\right.
\end{aligned}
$$

where $\zeta=x+y-(1 / 4) t$, and $\lambda, c_{1}$, and $c_{2}$ are arbitrary constants.

On the other hand, when setting $c_{1}=0$ and $c_{2}=1$, the solution of (23) can be written as

$$
v_{3}(\zeta)=0.25+0.25 i \cot \{0.25 i \zeta\} .
$$

Setting again $c_{2}=0$ and $c_{1}=1$, the solution of (23) can be written as

$$
v_{4}(\zeta)=0.25+0.25 i \tan \{0.25 i \zeta\}
$$


Case $c$. When $\lambda^{2}-4 \mu=0$, the rational function solutions of (8) are as follows:

$$
v(\zeta)=\frac{1}{2} \lambda+\frac{1}{4}+\left(\frac{c_{2}}{c_{1}+c_{2} \zeta}\right)-\frac{\lambda}{2}
$$

Then (26) becomes

$$
v_{5}=0.25+\frac{c_{2}}{c_{1}+\left(c_{2} x+c_{2} y-c_{2} t\right)}
$$

By $v=d u / d \zeta \rightarrow u=\int v$ and $w=\partial u / \partial x$, we find the all traveling wave solutions of (8) as follows:

$$
\begin{aligned}
u_{1}= & 0.25(x+y-0.25 t) \\
& -0.5 \ln (\operatorname{coth}\{0.25(x+y-0.25 t)\}-1 .) \\
& -0.5 \ln (\operatorname{coth}\{0.25(x+y-0.25 t)\}+1 .), \\
u_{2}= & 0.25(x+y-0.25 t) \\
& -0.5 \ln (\tanh \{0.25(x+y-0.25 t)\}-1 .) \\
& -0.5 \ln (\tanh \{0.25(x+y-0.25 t)\}+1 .), \\
u_{3}= & 0.25(x+y-0.25 t) \\
& +0.5 \ln (\operatorname{coth}\{0.25(x+y-0.25 t)\}-1 .) \\
& +0.5 \ln (\operatorname{coth}\{0.25(x+y-0.25 t)\}+1 .), \\
u_{4}= & 0.25(x+y-0.25 t) \\
& +0.5 \ln (\tanh \{0.25(x+y-0.25 t)\}-1 .) \\
& +0.5 \ln (\tanh \{0.25(x+y-0.25 t)\}+1 .),
\end{aligned}
$$

then we found

$$
\begin{aligned}
w_{1}= & 0.25-\frac{0.5\left(0.25-0.25 \operatorname{coth}\{-0.25 x-0.25 y+0.0625 t\}^{2}\right)}{-\operatorname{coth}\{-0.25 x-0.25 y+0.0625 t\}-1} \\
& -\frac{0.5\left(0.25-0.25 \operatorname{coth}\{-0.25 x-0.25 y+0.0625 t\}^{2}\right)}{-\operatorname{coth}\{-0.25 x-0.25 y+0.0625 t\}+1},
\end{aligned}
$$

$$
\begin{gathered}
w_{2}=0.25-\frac{0.5\left(0.25-0.25 \tanh \{-0.25 x-0.25 y+0.0625 t\}^{2}\right)}{-\tanh \{-0.25 x-0.25 y+0.0625 t\}-1} \\
-\frac{0.5\left(0.25-0.25 \tanh \{-0.25 x-0.25 y+0.0625 t\}^{2}\right)}{-\tanh \{-0.25 x-0.25 y+0.0625 t\}+1},
\end{gathered}
$$

$$
\begin{aligned}
w_{3}= & 0.25+\frac{0.5\left(0.25-0.25 \operatorname{coth}\{-0.25 x-0.25 y+0.0625 t\}^{2}\right)}{-\operatorname{coth}\{-0.25 x-0.25 y+0.0625 t\}-1} \\
& -\frac{0.5\left(0.25-0.25 \operatorname{coth}\{-0.25 x-0.25 y+0.0625 t\}^{2}\right)}{-\operatorname{coth}\{-0.25 x-0.25 y+0.0625 t\}+1},
\end{aligned}
$$

$$
\begin{aligned}
w_{4}= & 0.25+\frac{0.5\left(0.25-0.25 \tanh \{-0.25 x-0.25 y+0.0625 t\}^{2}\right)}{-\tanh \{-0.25 x-0.25 y+0.0625 t\}-1} \\
& -\frac{0.5\left(0.25-0.25 \tanh \{-0.25 x-0.25 y+0.0625 t\}^{2}\right)}{-\tanh \{-0.25 x-0.25 y+0.0625 t\}+1} .
\end{aligned}
$$

Similarly a second traveling wave solution set can be obtained by following the above procedure.

\section{Tanh Method}

In this paper, we used tanh method in its standard form presented by Malfliet [36, 37], and the outline of tanh method is known. For more details, refer to [38].

For computation the exact traveling wave solutions of equation generated by the Jaulent-Miodek Hierarchy. We used the ansatz

$$
S(Y)=\sum_{i=0}^{m} a_{i} Y^{i}
$$

where $Y=\tanh \{\mu \zeta\}$.

Substituting (33) in the ordinary differential equation of the equation generated by the Jaulent-Miodek Hierarchy, we found

$$
\begin{aligned}
16 c S & +4\left[\mu^{2}\left(1-Y^{2}\right)\left(-2 Y \frac{d s}{d y}+\left(1-Y^{2}\right) \frac{d^{2} s}{d y^{2}}\right)\right] \\
& -8 S^{3}+3 S+6 S^{2}=0 .
\end{aligned}
$$

To determine the parameter $m$, we usually balance the linear term of highest order in the resulting equation (34) with the highest order nonlinear terms, and we find $m=1$. This means that

$$
v(\zeta)=S(Y)=a_{0}+a_{1} Y
$$

Substituting $S(Y), S^{\prime}(Y), S^{\prime \prime}(Y), S^{2}(Y)$, and $S^{3}(Y)$ from (35) into (34) yields the system of algebraic equations for $a_{0}, a_{1}, c$, and $\mu$ as follows:

$$
\begin{aligned}
& Y^{0}:=(16 c+3) a_{0}-8 a_{0}^{3}+6 a_{0}^{2}=0, \\
& Y^{1}:=(16 c+3) a_{1}-8 \mu^{2} a_{1}-24 a_{0}^{2}+12 a_{0} a_{1}=0, \\
& Y^{2}: \quad=-24 a_{0} a_{1}^{2}+6 a_{1}^{2}=0, \\
& Y^{3}: \quad=-8 \mu^{2} a_{1}-8 a^{3}=0 .
\end{aligned}
$$




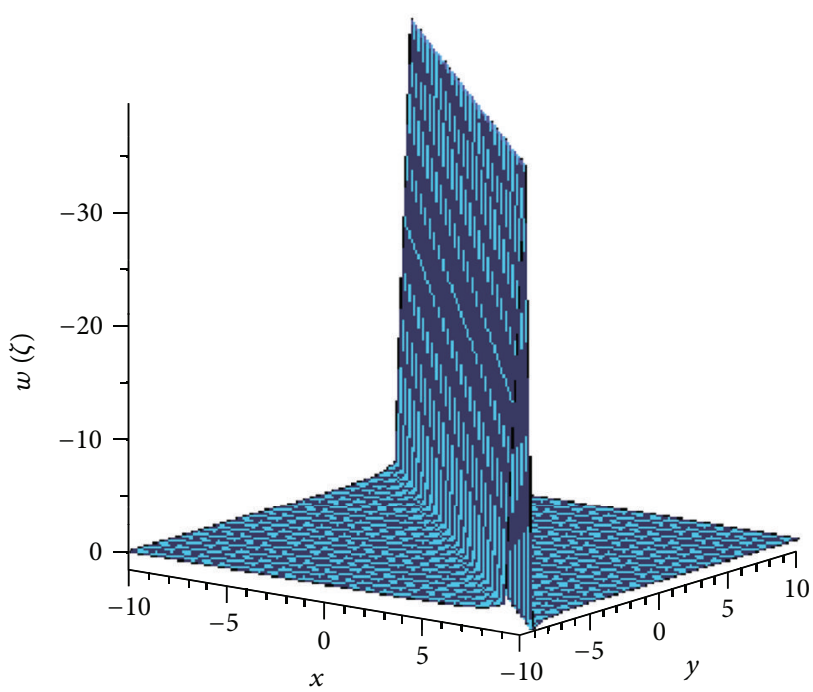

(a)

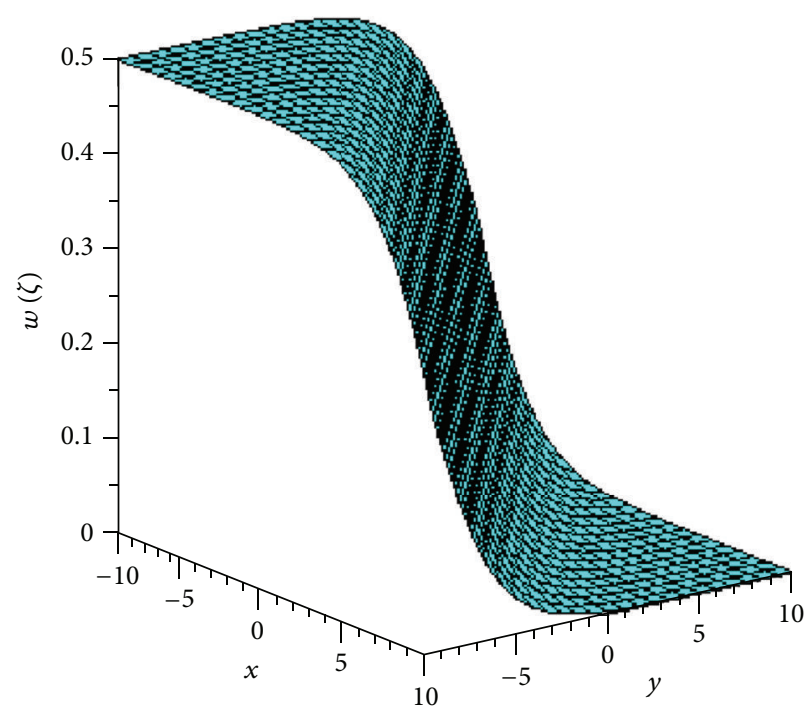

(c)

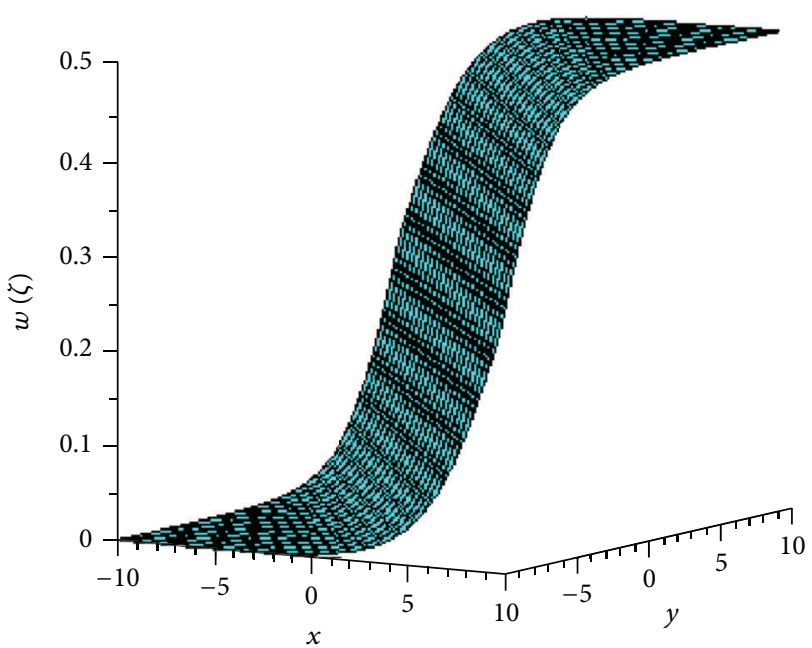

(b)

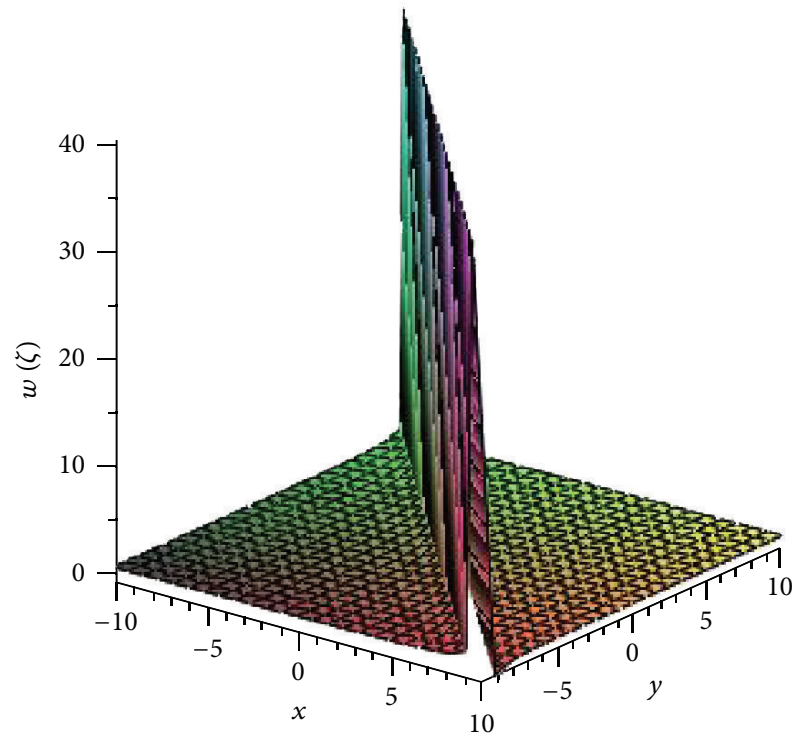

(d)

Figure 1: 3D plots of (a) (29), (b) (30), (c) (31), and (d) (32), for the parameter values $t=0.1$ and $c-1 / 4$.

Solving the system, we obtain

$$
\begin{aligned}
& a_{0}=\frac{1}{4}, \\
& a_{1}= \pm \frac{1}{4} \\
& c=-\frac{1}{4} \\
& \mu= \pm \frac{1}{4} .
\end{aligned}
$$

In view of (37), we obtain the solitary wave solutions

$$
\begin{gathered}
v(x, y, t)=0.25+0.25 \tanh \{0.25 \zeta\} \\
v(x, y, t)=0.25-0.25 \tanh \{-0.25 \zeta\}
\end{gathered}
$$

where $\zeta=x+y-0.25 t$.

Equation (38) is the same as the result obtained by the $\left(G^{\prime} / G\right)$-expansion method.

We obtain the behaviour of exact traveling wave solution of (8). 


\section{Conclusion}

In this work, we have established the traveling wave solutions of the nonlinear equation generated by the Jaulent-Miodek Hierarchy. It appears that three types of traveling wave solutions have been successfully found by using the $\left(G^{\prime} / G\right)$ expansion method. The general traveling wave solutions are hyperbolic function solutions, trigonometric function solutions, and rational function solutions. The solitary wave solutions are derived from these functions when the parameters are taken as special values. The solutions of the equation via $\left(G^{\prime} / G\right)$-expansion method are exactly the same as those obtained by tanh method if the condition $c_{2}=0$ is satisfied, this means that the tanh method is a special case of $\left(G^{\prime} / G\right)$ expansion method. The $\left(G^{\prime} / G\right)$-expansion method is quite efficient and practically well suited for use in finding the exact solutions for the equation generated by the JaulentMiodek Hierarchy. Some solutions given in this paper are new solutions which have not been reported yet. Threedimensional plots (Figure 1) of some of the investigated solutions are also drawn to visualize the underlying dynamics of such results. The method which we have proposed in this work is also a standard, direct, and computerizable method.

\section{References}

[1] A.-M. Wazwaz, "Multiple kink solutions and multiple singular kink solutions for $(2+1)$-dimensional nonlinear models generated by the Jaulent-Miodek hierarchy," Physics Letters A, vol. 373, no. 21, pp. 1844-1846, 2009.

[2] H. Liu and F. Yan, "The bifurcation and exact travelling wave solutions for $(2+1)$-dimensional nonlinear models generated by the Jaulent-Miodek hierarchy," International Journal of Nonlinear Science, vol. 11, no. 2, pp. 200-205, 2011.

[3] A.-M. Wazwaz, "The tanh-coth and the sine-cosine methods for kinks, solitons, and periodic solutions for the PochhammerChree equations," Applied Mathematics and Computation, vol. 195, no. 1, pp. 24-33, 2008.

[4] A.-M. Wazwaz, "Abundant solitons solutions for several forms of the fifth-order KdV equation by using the tanh method," Applied Mathematics and Computation, vol. 182, no. 1, pp. 283300, 2006.

[5] A.-M. Wazwaz, "The tanh method for traveling wave solutions of nonlinear equations," Applied Mathematics and Computation, vol. 154, no. 3, pp. 713-723, 2004.

[6] A.-M. Wazwaz, "The sine-cosine and the tanh methods: reliable tools for analytic treatment of nonlinear dispersive equations," Applied Mathematics and Computation, vol. 173, no. 1, pp. 150$164,2006$.

[7] M. L. Wang, "Solitary wave solutions for variant Boussinesq equations," Physics Letters A, vol. 199, no. 3-4, pp. 169-172, 1995.

[8] M. A. Noor, S. T. Mohyud-Din, A. Waheed, and E. A. Al-Said, "Exp-function method for traveling wave solutions of nonlinear evolution equations," Applied Mathematics and Computation, vol. 216, no. 2, pp. 477-483, 2010.

[9] S. T. Mohyud-Din, M. A. Noor, and K. I. Noor, "Expfunction method for traveling wave solutions of modified ZakharovKuznetsov equation," Journal of King Saud University, vol. 22, no. 4, pp. 213-216, 2010.

[10] A.-M. Wazwaz, "Multiple-soliton solutions for the KP equation by Hirota's bilinear method and by the tanh-coth method,"
Applied Mathematics and Computation, vol. 190, no. 1, pp. 633640, 2007.

[11] A.-M. Wazwaz, "The Hirota's direct method for multiple-soliton solutions for three model equations of shallow water waves," Applied Mathematics and Computation, vol. 201, no. 1-2, pp. 489-503, 2008.

[12] G. Ebadi, N. Yousefzadeh, H. Triki, and A. Yildirim, "Envelope solitons, periodic wave and other solutions to BoussinesqBurgers equation," Romanian Reports in Physics, vol. 64, no. 4, pp. 915-932, 2012.

[13] A. Biswas and A. H. Kara, "1-soliton solution and conservation laws for the Jaulent-Miodek equation with power law nonlinearity," Applied Mathematics and Computation, vol. 217, no. 2, pp. 944-948, 2010.

[14] M. Wang, X. Li, and J. Zhang, "The $\left(G^{\prime} / G\right)$-expansion method and travelling wave solutions of nonlinear evolution equations in mathematical physics," Physics Letters A, vol. 372, no. 4, pp. 417-423, 2008.

[15] A. Bekir, "Application of the $\left(G^{\prime} / G\right)$-expansion method for nonlinear evolution equations," Physics Letters A, vol. 372, no. 19, pp. 3400-3406, 2008.

[16] J. Zhang, X. Wei, and Y. Lu, "A generalized $\left(G^{\prime} / G\right)$-expansion method and its applications," Physics Letters A, vol. 372, no. 20, pp. 3653-3658, 2008.

[17] S. Zhang, J. L. Tong, and W. Wang, "A generalized $\left(G^{\prime} / G\right)$ expansion method for the $\mathrm{mKdV}$ equation with variable coefficients," Physics Letters A, vol. 372, no. 13, pp. 2254-2257, 2008.

[18] G. Ebadi, A. H. Kara, M. D. Petkovic, A. Yildirim, and A. Biswas, "Solitons and conserved quantities of the Ito equation," Proceedings of the Romanian Academy A, vol. 13, no. 3, pp. 215224, 2012.

[19] B. Ayhan and A. Bekir, "The $\left(G^{\prime} / G\right)$-expansion method for the nonlinear lattice equations," Communications in Nonlinear Science and Numerical Simulation, vol. 17, no. 9, pp. 3490-3498, 2012.

[20] A. Malik, F. Chand, H. Kumar, and S. C. Mishra, "Exact solutions of the Bogoyavlenskii equation using the multiple $\left(G^{\prime} / G\right)$-expansion method," Computers \& Mathematics with Applications, vol. 64, no. 9, pp. 2850-2859, 2012.

[21] H. Jafari, N. Kadkhoda, and A. Biswas, "The $\left(G^{\prime} / G\right)$-expansion method for solutions of evolution equations from isothermal magnetostatic atmospheres," Journal of King Saud University Science, vol. 25, no. 1, pp. 47-62, 2013.

[22] E. J. Parkes, “Comment on application of $\left(G^{\prime} / G\right)$-expansion method to travelling wave solutions of three nonlinear evolution equation [comput fluids 2010,39:195763]," Computers \& Fluids, vol. 42, no. 1, pp. 108-109, 2011.

[23] B. Zheng, "Travelling wave solutions of two nonlinear evolution equations by using the $\left(G^{\prime} / G\right)$-expansion method," Applied Mathematics and Computation, vol. 217, no. 12, pp. 5743-5753, 2011.

[24] J. Feng, W. Li, and Q. Wan, "Using $\left(G^{\prime} / G\right)$-expansion method to seek the traveling wave solution of Kolmogorov-PetrovskiiPiskunov equation," Applied Mathematics and Computation, vol. 217, no. 12, pp. 5860-5865, 2011.

[25] M. M. Kabir, A. Borhanifar, and R. Abazari, "Application of $\left(G^{\prime} / G\right)$-expansion method to regularized long wave (RLW) equation," Computers \& Mathematics with Applications, vol. 61, no. 8, pp. 2044-2047, 2011. 
[26] J.-M. Zuo and Y.-M. Zhang, "Application of the $\left(G^{\prime} / G\right)$ expansion method to solve coupled MKdV equations and coupled Hirota-Satsuma coupled KdV equations," Applied Mathematics and Computation, vol. 217, no. 12, pp. 5936-5941, 2011.

[27] N. A. Kudryashov and P. N. Ryabov, "Comment on: application of the $\left(G^{\prime} / G\right)$ method for the complex KdV equation [Huiqun Zhang, Commun Nonlinear Sci Numer Simul 15;2010:17001704]," Communications in Nonlinear Science and Numerical Simulation, vol. 16, no. 1, pp. 596-598, 2011.

[28] T. Özis and I. Aslan, "Application of the $\left(G^{\prime} / G\right)$-expansion method to Kawahara type equations using symbolic computation," Applied Mathematics and Computation, vol. 216, no. 8, pp. 2360-2365, 2010.

[29] G. Ebadi and A. Biswas, "Application of the $\left(G^{\prime} / G\right)$-expansion method for nonlinear diffusion equations with nonlinear source," Journal of the Franklin Institute, vol. 347, no. 7, pp. 13911398, 2010.

[30] M. Hayek, "Constructing of exact solutions to the $\mathrm{KdV}$ and Burgers equations with power-law nonlinearity by the extended $\left(G^{\prime} / G\right)$-expansion method," Applied Mathematics and Computation, vol. 217, no. 1, pp. 212-221, 2010.

[31] H. Zhang, "Application of the $\left(G^{\prime} / G\right)$-expansion method for the complex KdV equation," Communications in Nonlinear Science and Numerical Simulation, vol. 15, no. 7, pp. 1700-1704, 2010.

[32] Z.-L. Li, "Constructing of new exact solutions to the GKdVmKdV equation with any-order nonlinear terms by $\left(G^{\prime} / G\right)$ expansion method," Applied Mathematics and Computation, vol. 217, no. 4, pp. 1398-1403, 2010.

[33] A. Malik, F. Chand, and S. C. Mishra, "Exact travelling wave solutions of some nonlinear equations by $\left(G^{\prime} / G\right)$-expansion method," Applied Mathematics and Computation, vol. 216, no. 9, pp. 2596-2612, 2010.

[34] A. R. Shehata, "The traveling wave solutions of the perturbed nonlinear Schrödinger equation and the cubic-quintic Ginzburg Landau equation using the modified $\left(G^{\prime} / G\right)$-expansion method," Applied Mathematics and Computation, vol. 217, no. 1, pp. 1-10, 2010.

[35] S. Kutluay, A. Esen, and O. Tasbozan, “The $\left(G^{\prime} / G\right)$-expansion method for some nonlinear evolution equations," Applied Mathematics and Computation, vol. 217, no. 1, pp. 384-391, 2010.

[36] W. Malfliet, "Solitary wave solutions of nonlinear wave equations," American Journal of Physics, vol. 60, no. 7, pp. 650-654, 1992.

[37] W. Malfliet and W. Hereman, "The tanh method. II. Perturbation technique for conservative systems," Physica Scripta, vol. 54, no. 6, pp. 569-575, 1996.

[38] A. J. M. Jawad, M. D. Petkovic, and A. Biswas, "Soliton solutions of a few nonlinear wave equations," Applied Mathematics and Computation, vol. 216, no. 9, pp. 2649-2658, 2010. 


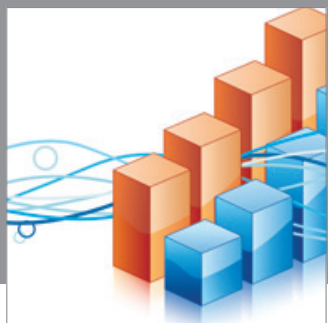

Advances in

Operations Research

mansans

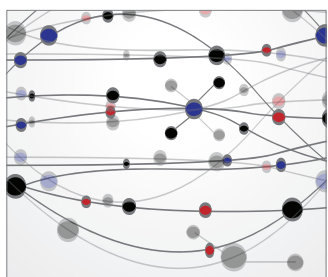

The Scientific World Journal
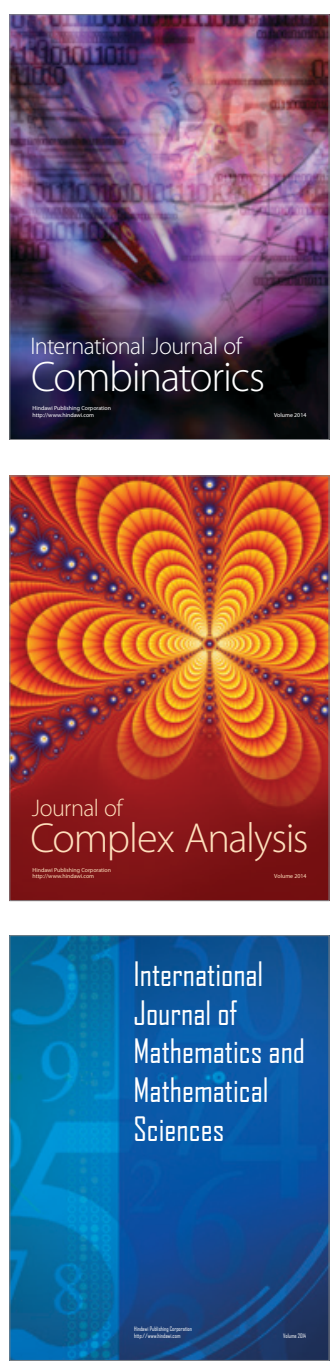
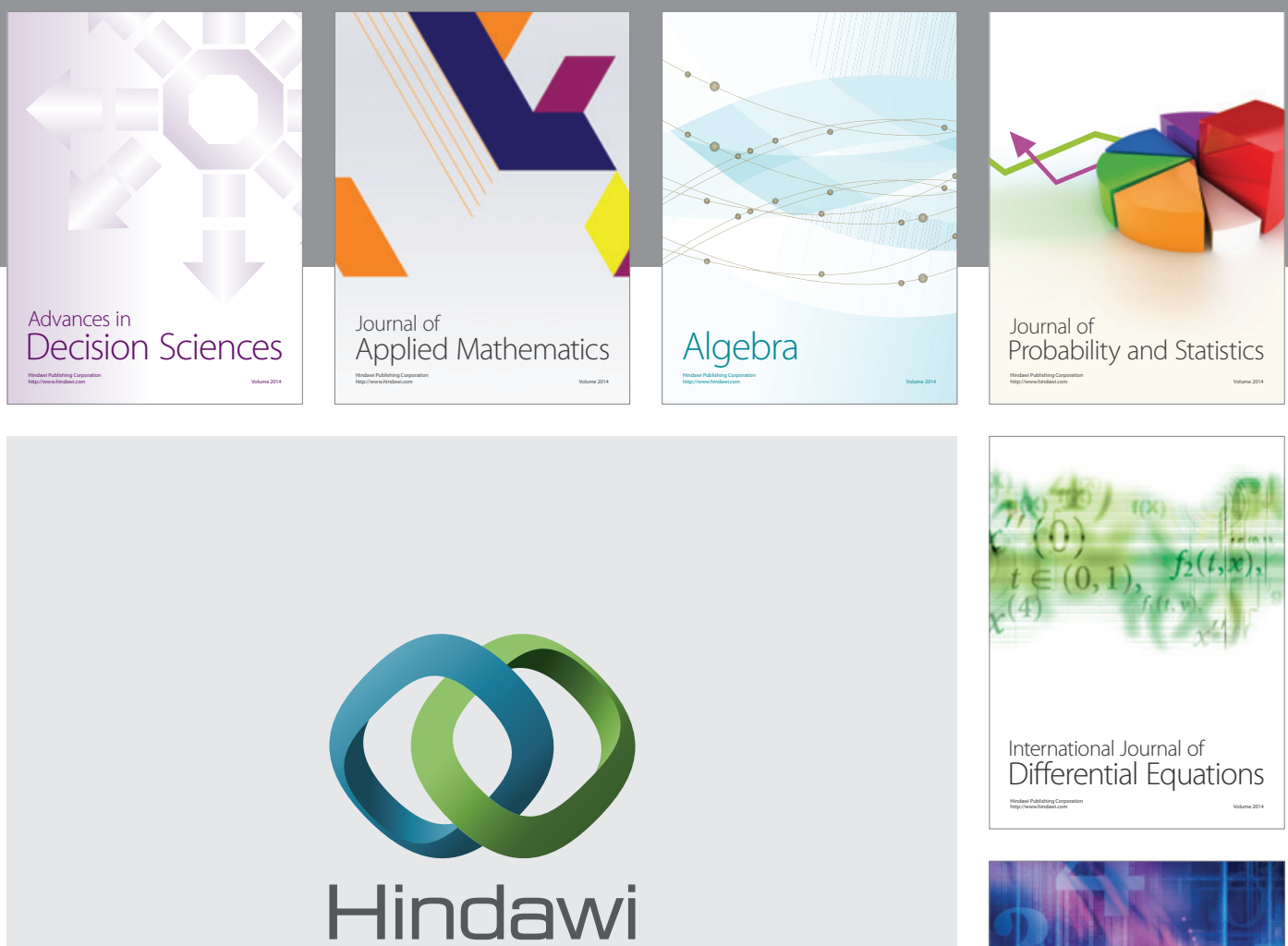

Submit your manuscripts at http://www.hindawi.com
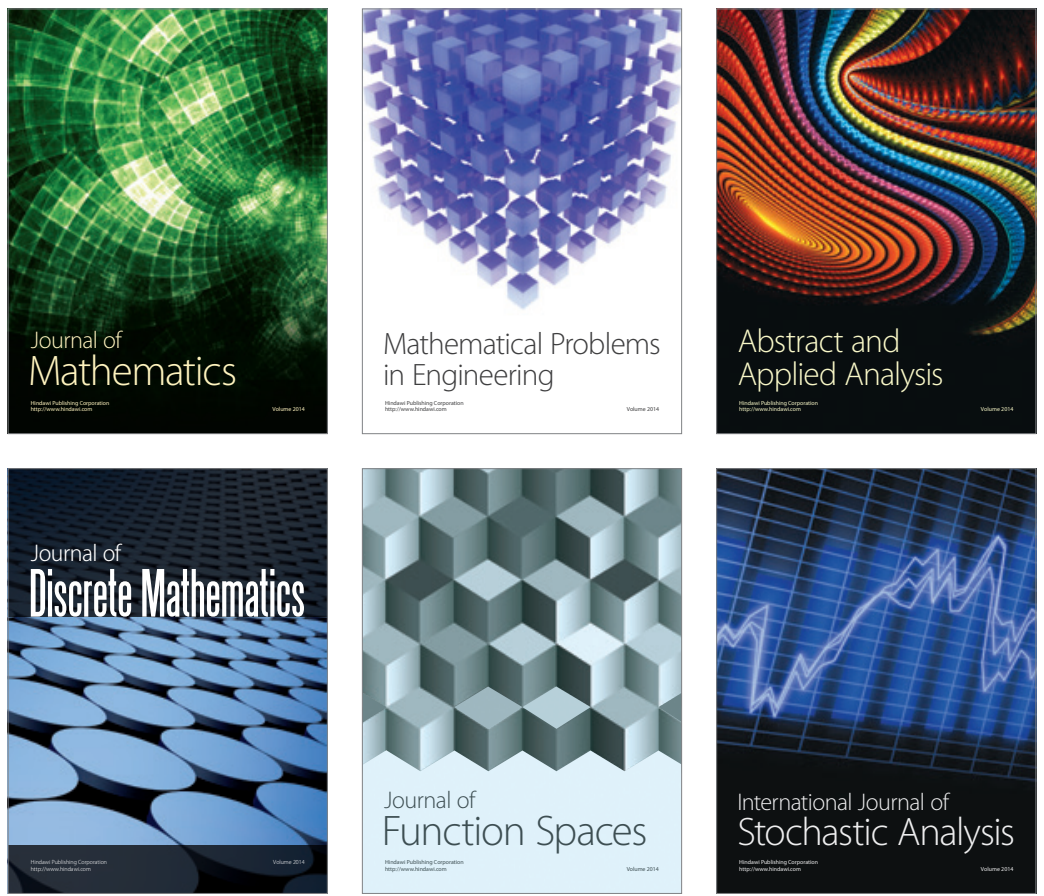

Journal of

Function Spaces

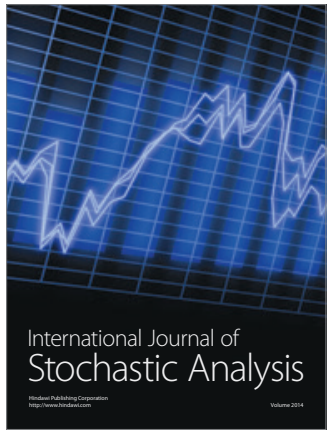

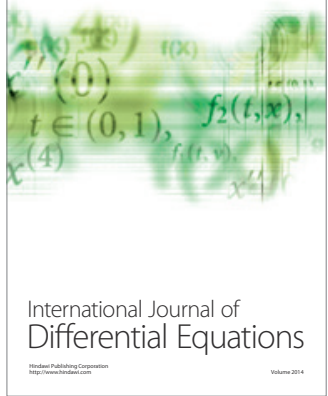
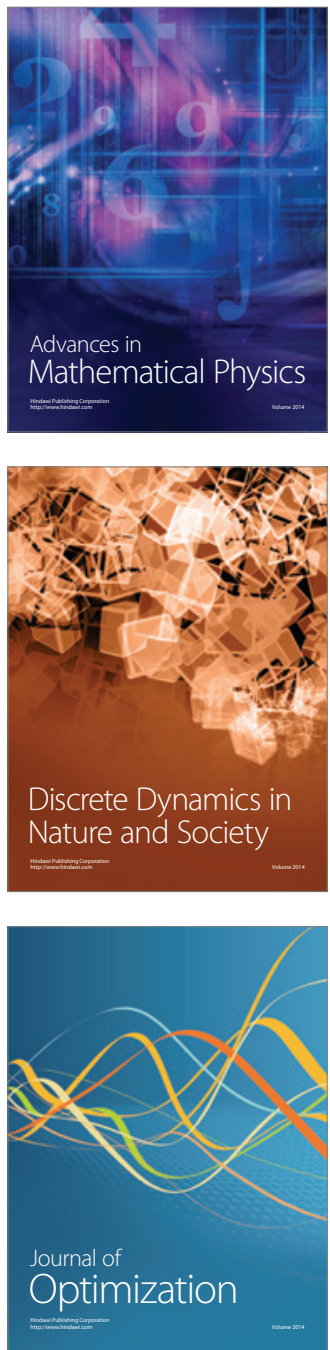